Research article

Open Access

\title{
Synthesis by Ring-Closing Metathesis and Cytotoxic Evaluation of Novel Thienylmacrolactones
}

\author{
Jürgen Krauss *, Daniela KöbleR, Verena Miller, Franz Bracher
}

Department of Pharmacy - Center for Drug Research, Ludwig-Maximilians University, Butenandtstr. 5-13, 81377 Munich, Germany.

* Corresponding author. E-mail: hjkra@cup.uni-muenchen.de (J. Krauss)

Sci Pharm. 2012; 80: 29-36

doi:10.3797/scipharm.1109-09

Published: November $1^{\text {st }} 2011 \quad$ Received: $\quad$ September $17^{\text {th }} 2011$

Accepted: $\quad$ November $1^{\text {st }} 2011$

This article is available from: http://dx.doi.org/10.3797/scipharm.1109-09

(c) Krauss et al.; licensee Österreichische Apotheker-Verlagsgesellschaft m. b. H., Vienna, Austria.

This is an Open Access article distributed under the terms of the Creative Commons Attribution License (http://creativecommons.org/licenses/by/3.0/), which permits unrestricted use, distribution, and reproduction in any medium, provided the original work is properly cited.

\begin{abstract}
This paper describes the synthesis and biological evaluation of macrolactones containing a thienyl substituent as simple analogues of epothilones. The compounds were prepared in a brief and efficient manner from thiophene-2carbaldehyde using a ring-closing metathesis with Grubbs I or Grubbs II catalyst as the key step. The target lactones showed only insignificant cytotoxicity, while an intermediate simple thienyl carbinol showed very promising cytotoxicity.
\end{abstract}

\section{Keywords}

Ring-closing metathesis $\bullet$ Grignard reaction $\bullet$ Macrolactones $\bullet$ MTT assay $\bullet$ Thiophenes

\section{Introduction}

The macrolactone scaffold is found in numerous natural products $[1,2]$ which show a wide range of pharmacological properties including antimicrobial and cytotoxic activities. Prominent examples are the 14-membered lactone antibiotics erythromycin and telithromycin, as well as their 15-membered, semisynthetic derivative azithromycin [3], the cytotoxic salicylihalamides (12-membered lactones) [4,5], the 18-membered cytotoxic antibiotic FD-891 [6] and the epothilones, cytotoxic 16-membered lactones which inhibit microtubule function [7]. Extensive investigations on total syntheses [8] and analysis of structure-activity relationships of epothilones have been performed in the past, and a number of substances from this class have entered clinical studies [9]. Sagopilone, a new clinical candidate from the epothilone family [10], demonstrates that the rather complex 
methylthiazolylpropenyl residue of native epothilones can be replaced by a simple heteroaromatic residue, in this case a benzothiazole.

This prompted us to investigate a new type of 16-membered lactones bearing a sulphurcontaining hetarene, namely a thiophene ring [11], next to the lactone oxygen in analogy to new synthetic epothilones. The target compounds were to be prepared using the ringclosing metathesis methodology we worked out earlier for various furyl macrolactones $[12,13]$.<smiles>COC(CC1OC1(C)CCC[C@H](C)[C@@H](O)[C@@H](O)C(=O)C(C)(C)[C@H](O)CC(=O)O)/C(C)=C/c1csc(C)n1</smiles>

I<smiles>C=CC[C@H](C(=O)C(C)(C)[C@@H](O)CC(=O)OC(CC1OC1(C)CCCCC(C)C)c1ccc2sc(C)nc2c1)C(C)O</smiles>

II

Fig. 1. Structures of epothilone B (I) and sagopilone (II)

\section{Results and Discussion}

Thiophene-2-carbaldehyde (1) was reacted in a Grignard reaction with pent-4-enylmagnesium bromide to give alcohol 2, following a methodology we had worked out earlier for furan derivatives $[14,15]$. Secondary alcohol 2 was esterified with undec-10-enoyl chloride to give the ester 3 . From this intermediate containing two terminal vinyl groups the macrolactone $\mathbf{4}$ was prepared in a ring-closing metathesis under high dilution conditions using either Grubbs I catalyst, benzylidene(dichloro)bis(tricyclohexylphosphane)ruthenium, or Grubbs II catalyst, benzylidene[1,3-bis(2,4,6-trimethylphenyl) 2-imidazolidinylidene]dichloro(tricyclohexylphosphane)ruthenium. When using Grubbs II catalyst, the reaction proceeded faster than with Grubbs I catalyst ( 6 h vs. $24 \mathrm{~h}$ ), for comparable observations see ref. $[12,16,17]$.

Both $1^{\text {st }}$ and $2^{\text {nd }}$ generation Grubbs catalysts gave the expected 16-membered lactone 4 as $E / Z$ mixtures (Grubbs I catalyst: $E / Z=66: 34$; Grubbs II catalyst: $E / Z=77: 23$ ). The $E / Z$ isomers could not be separated by flash column chromatography. The ratio of the isomers was determined by GLC-MS and NMR spectroscopy. The predominating E-isomer was identified by the vicinal coupling constant of both olefinic protons of ${ }^{3} \mathrm{~J}=15.3 \mathrm{~Hz}$. Since both olefinic hydrogens showed the same chemical shift, the signal and the coupling constants were verified by computer-aided techniques (NutsPro - NMR Utility Transform Software - Professional, 2D Professional Version - 20020107). The simulated ${ }^{1} \mathrm{H}-\mathrm{NMR}$ signal and the simulated coupling constants for the olefinic protons of the $E$-isomer were in full accordance to the measured signal and coupling constants (Fig. 2). 


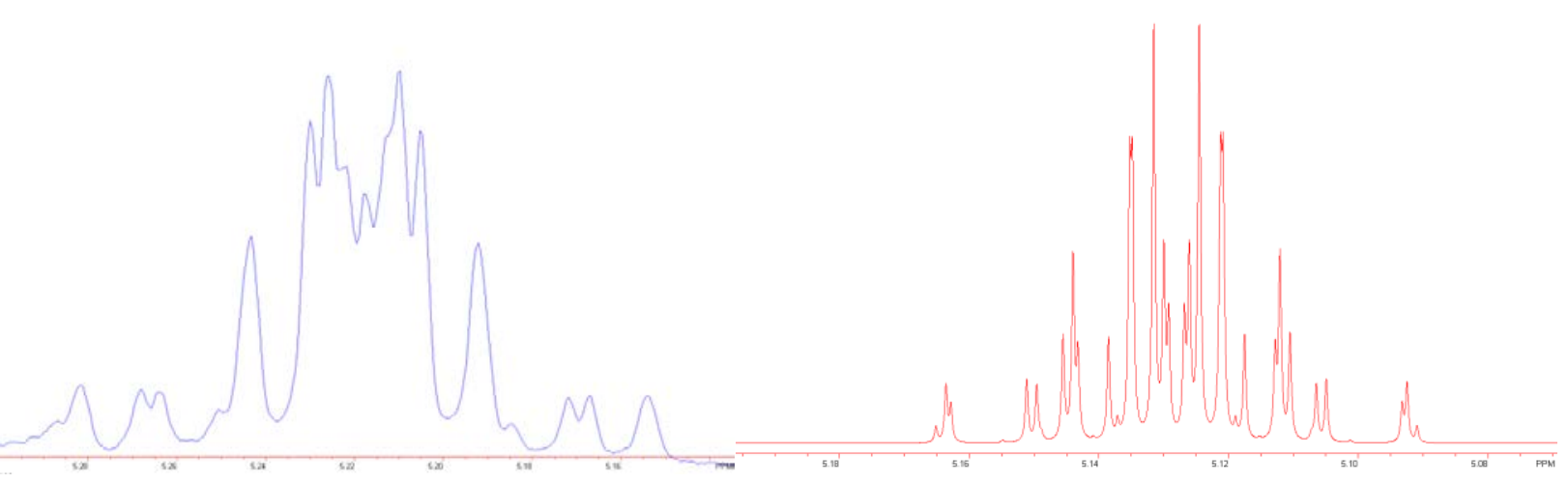

Fig. 2. ${ }^{1} \mathrm{H}$-NMR resonances of the olefinic protons of the $E$-configurated product 4 (left), and simulated ${ }^{1} \mathrm{H}$-NMR resonances for the $E$-isomer with ${ }^{3} \mathrm{~J}=15.3 \mathrm{~Hz}$ for the coupling of the olefinic protons and ${ }^{3} \mathrm{~J}=7.0 \mathrm{~Hz}$ for the coupling of the olefinic protons with the neighboring methylene protons.

Since in preliminary screenings lactone $\mathbf{4}$ showed negligible cytotoxicity, we intended to introduce hydroxy groups into the ring system to enhance structural similarity to the epothilones. The olefinic double bond of the $E / Z$ mixture 4 could be dihydroxylated using a Sharpless dihydroxylation with $\beta$-AD-mix ${ }^{\circledR}\left(\mathrm{OsO}_{4}, \mathrm{~K}_{3} \mathrm{Fe}(\mathrm{CN})_{6}, \mathrm{~K}_{2} \mathrm{CO}_{3},(\mathrm{DHQD})_{2}-\mathrm{PHAL}\right)$ $[14,18]$ to give an inseparable mixture of the isomeric diols 5 .<smiles>O=Cc1cccs1</smiles>

1

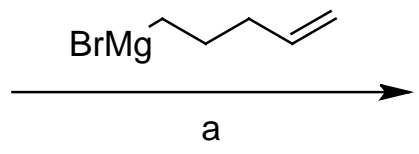<smiles>C=CCCCC(O)c1cccs1</smiles>

2<smiles>C=CCCCCCCCCC(=O)Cl</smiles><smiles>C=CCCCCCCCCCC(=O)OC(CCCC=C)c1cccs1</smiles>

3<smiles>CCCCCC(OC(=O)CCCCCCCC/C=C\CCCCCC(C)(C)C)c1cccs1</smiles>

4<smiles>O=C(O)CCCCCCCCC(O)C1CCCCC1OC(O)c1cccs1</smiles>

5

Sch. 1. a: THF. b: toluene, EDMA. c: toluene, Grubbs catalyst $\left(1^{\text {st }}\right.$ or $2^{\text {nd }}$ generation $)$, high dilution technique. d: tert.-butanol / $\mathrm{H}_{2} \mathrm{O}, \beta-A D$ mix $^{\circledR}$. 
The resulting lactones as well as the precursors $\mathbf{2}$ and $\mathbf{3}$ were tested in an agar diffusion assay against several bacteria and fungi. The compounds showed no significant antimicrobial activities compared to tetracycline or clotrimazol.

The cytotoxicity was determined in a MTT assay on HL-60 cells (human leukaemia cell line) using the method of Mosmann [19]. The macrolactones $\mathbf{4}$ and $\mathbf{5}$ exhibited only extremely weak cytotoxic activity. Only the secondary alcohol $\mathbf{2}$ showed high cytotoxic activity in the low $\mu \mathrm{M}$ range (Tab. 1). Compound 5 was also tested in the $\mathrm{NCl}$ single high dose cell panel assays on 59 cell lines, but showed no remarkable selectivity or cytotoxicity in this assay.

Tab. 1. Cytotoxicity against HL 60 cells.

\begin{tabular}{ccc}
\hline compound & $\begin{array}{c}\mathrm{IC}_{50} \\
{[\mu \mathrm{M}(\boldsymbol{\mu g} / \mathrm{mL})]}\end{array}$ & $\begin{array}{c}\log \mathbf{P} \\
\text { (calcd.) }\end{array}$ \\
\hline $\mathbf{2}$ & $6(1.1)$ & 3.2 \\
$\mathbf{3}$ & $>100$ & 7.2 \\
$\mathbf{4}$ & $290(92.8)$ & 6.1 \\
$\mathbf{5}$ & $120(42.5)$ & 4.2 \\
cisplatin & $5(1.5)$ & \\
\hline
\end{tabular}

\section{Conclusion}

In conclusion, we worked out a short and efficient synthesis of novel thienyl macrolactones related to the epothilones. The resulting lactones $\mathbf{4}$ and $\mathbf{5}$ did not show significant anticancer or antimicrobial activities.

We supposed that a reason for the negligible cytotoxic activity of lactone 4 might be its very high lipophilicity, as shown by the calculated $\log P$ value (Tab. 1). The $\log P$ of 7.2 is much higher than the log $P$ limits defined by Lipinski $(-0.4$ to +5.6$)[20]$ for drug likeness. In fact, the diol 5 (calculated log $P=4.2$ ) showed increased cytotoxicity compared to 4 , but still is far away from being an interesting lead. To our great surprise, the secondary thienyl carbinol 2 showed very interesting cytotoxic activity $\left(\mathrm{IC}_{50}=6 \mu \mathrm{M}\right)$. This reminds us of an accidental observation in previous work [12] where a side-product containing a furyl carbinol showed comparable cytotoxicity.

Work is in progress to gain deeper insight into structure-activity relationships of the interesting class of heteroaryl carbinols and to identify their molecular mode of action.

\section{Experimental}

\section{General}

Elemental analysis: Heraeus CHN-Rapid; IR-Spectra: Perkin-Elmer FT-IR Paragon 1000; MS: Hewlett Packard MS-Engine; electron ionisation (El) $70 \mathrm{eV}$, chemical ionisation (CI) with $\mathrm{CH}_{4}(300 \mathrm{eV})$; NMR: Jeol GSX $400\left({ }^{1} \mathrm{H}: 400 \mathrm{MHz},{ }^{13} \mathrm{C}: 100 \mathrm{MHz}\right)$; melting points: Büchi Melting Point B-540 (not corrected); flash column chromatography (FCC): silica gel 60 (230-400 mesh, E. Merck, Darmstadt); GLC-MS: Shimadzu GC-17 A (carrier: He, oven 
temperature program: $100-280{ }^{\circ} \mathrm{C}, 10{ }^{\circ} \mathrm{C} / \mathrm{min}$, capillary column: Varian VF-5ms $30 \mathrm{~m} \times$ 0.25 , split injector $\mathrm{T}=250^{\circ} \mathrm{C}$, detector $\mathrm{T}=260{ }^{\circ} \mathrm{C}$ ).

\section{(士)-1-(Thiophen-2-yl)-hex-5-en-1-ol (2)}

To a mixture of $1.53 \mathrm{~g}(66.5 \mathrm{mmol})$ magnesium turnings and an iodine crystal in dry THF $(25 \mathrm{~mL})$ was added $6.60 \mathrm{~g}(44.3 \mathrm{mmol})$ 1-bromopent-4-ene in two portions. The mixture was heated until the Grignard reaction started. The suspension was heated under reflux for further $1 \mathrm{~h}$.

To a solution of $3.30 \mathrm{~g}$ (29.5 mmol) thiophene-2-carbaldehyde (1) in $15 \mathrm{ml}$ dry THF the prepared solution of pent-4-enylmagnesium bromide in THF was added dropwise. The mixture was stirred for $4 \mathrm{~h}$, than quenched with $30 \mathrm{~mL} 5 \%$ aqueous $\mathrm{NH}_{4} \mathrm{Cl}$ solution and extracted with diethyl ether $(3 \times 30 \mathrm{~mL})$. The combined organic layers were dried over $\mathrm{Na}_{2} \mathrm{SO}_{4}$ and the solvent was evaporated. The residue was purified by $\mathrm{FCC}$ (n-hexane/ethyl acetate $5: 1)$ to give $4.30 \mathrm{~g}(80 \%)$ of 2 as a colourless oil. ${ }^{1} \mathrm{H}-\mathrm{NMR}\left(400 \mathrm{MHz}, \mathrm{CDCl}_{3}\right)$ : $\delta$ $(\mathrm{ppm})=1.42(\mathrm{~m}, 1 \mathrm{H}, 3-\mathrm{H}), 1.55(\mathrm{~m}, 1 \mathrm{H}, 3-\mathrm{H}), 1.85(\mathrm{~m}, 2 \mathrm{H}, 2-\mathrm{H}), 2.09(\mathrm{~m}, 2 \mathrm{H}, 4-\mathrm{H}), 2.16$ $(\mathrm{s}, 1 \mathrm{H}, \mathrm{OH}), 4.90(\mathrm{t}, \mathrm{J}=6.5 \mathrm{~Hz}, 1 \mathrm{H}, 1-\mathrm{H}), 4.95(\mathrm{~m}, 1 \mathrm{H}, 6-\mathrm{H}), 5.01(\mathrm{~m}, 1 \mathrm{H}, 6-\mathrm{H}), 5.79(\mathrm{~m}$, $1 \mathrm{H}, 5-\mathrm{H}), 6.95\left(\mathrm{~m}, 2 \mathrm{H}, 2^{-}-\mathrm{H}, 3^{-}-\mathrm{H}\right), 7.23\left(\mathrm{~m}, 1 \mathrm{H}, 4^{\prime}-\mathrm{H}\right) .{ }^{13} \mathrm{C}-\mathrm{NMR}\left(400 \mathrm{MHz}, \mathrm{CDCl}_{3}\right): \delta$ $(\mathrm{ppm})=25.0(\mathrm{C}-3), 33.4(\mathrm{C}-4), 38.7(\mathrm{C}-2), 70.2$ (C-1), 114.8 (C-6), 123.7 (C-5), 124.5 (C3'), 126.6 (C-4'), 138.4 (C-5), 148.8 (C-2'). El-MS m/z (rel. int.): $182\left(\mathrm{M}^{+}, 100\right), 164\left(\mathrm{M}^{+}-\right.$ 18, 30), 97 (75). IR ( NaCl, film): v $\left[\mathrm{cm}^{-1}\right]=3379,3074,2936,1860,1640,1439,1414$, 1066, 1029, 995, 912, 852, 830, 699. Elemental analysis: $\mathrm{C}_{10} \mathrm{H}_{14} \mathrm{OS}$ (182.29). Calcd.: C: 65.89. H: 7.74. S: 17.59. Found: C: 65.71 . H: 7.91. S: 17.17.

\section{(士)-1-(Thiophen-2-yl)hex-5-en-1-yl undec-10-enoate (3)}

$1.60 \mathrm{~g}$ (8.80 mmol) of 2 were dissolved in $30 \mathrm{~mL}$ dry toluene, $2.10 \mathrm{~g}$ (10.6 mmol) undec10-enoyl chloride and $5 \mathrm{~mL} N$-ethyl- $N, N$-dimethylamine (EDMA) were added. The mixture was stirred for $5 \mathrm{~h}$ and then the solvent was evaporated. The residue was dispersed in $20 \mathrm{~mL}$ water and extracted with diethyl ether $(3 \times 30 \mathrm{~mL})$. The combined organic layers were dried over $\mathrm{Na}_{2} \mathrm{SO}_{4}$ and the solvent was evaporated. The residue was purified by FCC (n-hexane/ethyl acetate 10:1 to $5: 1$ ) to give $1.85 \mathrm{~g}(60 \%)$ of 3 as a colourless oil. ${ }^{1} \mathrm{H}-\mathrm{NMR}$ $\left(400 \mathrm{MHz}, \mathrm{CDCl}_{3}\right): \delta(\mathrm{ppm})=1.26\left(\mathrm{~m}, 8 \mathrm{H}, 4 \mathrm{CH}_{2}\right), 1.36\left(\mathrm{~m}, 4 \mathrm{H}, 2 \mathrm{CH}_{2}\right), 1.58\left(\mathrm{~m}, 2 \mathrm{H}, \mathrm{CH}_{2}\right)$, $1.89\left(\mathrm{~m}, 1 \mathrm{H}, \mathrm{CH}_{2}\right), 2.03\left(\mathrm{~m}, 3 \mathrm{H}, \mathrm{CH}_{2}, \mathrm{CH}_{2}\right), 2.09\left(\mathrm{~m}, 2 \mathrm{H}, \mathrm{CH}_{2}\right), 2.30(\mathrm{t}, \mathrm{J}=8.0 \mathrm{~Hz}, 2 \mathrm{H}$, $\left.\mathrm{CH}_{2}\right), 4.96\left(\mathrm{~m}, 4 \mathrm{H}, 2=\mathrm{CH}_{2}\right), 5.79(\mathrm{~m}, 2 \mathrm{H}, 2-\mathrm{CH}=), 6.04(\mathrm{t}, \mathrm{J}=7.4 \mathrm{~Hz}, 1 \mathrm{H}, \mathrm{CH}), 6.95(\mathrm{dd}, \mathrm{J}$ $=4.9 \mathrm{~Hz}, \mathrm{~J}=3.4 \mathrm{~Hz}, 1 \mathrm{H}$, aromat. $\mathrm{CH}), 7.03(\mathrm{~d}, \mathrm{~J}=3.4 \mathrm{~Hz}, 1 \mathrm{H}$, aromat. $\mathrm{CH}), 7.25(\mathrm{dd}, \mathrm{J}=$ $4.9 \mathrm{~Hz}, \mathrm{~J}=1.1 \mathrm{~Hz}, 1 \mathrm{H}$, aromat. $\mathrm{CH}) .{ }^{13} \mathrm{C}-\mathrm{NMR}\left(400 \mathrm{MHz}, \mathrm{CDCl}_{3}\right): \delta(\mathrm{ppm})=24.8\left(\mathrm{CH}_{2}\right)$, $24.9\left(\mathrm{CH}_{2}\right), 28.9\left(\mathrm{CH}_{2}\right), 29.0\left(\mathrm{CH}_{2}\right), 33.2\left(\mathrm{CH}_{2}\right), 33.8\left(\mathrm{CH}_{2}\right), 34.5\left(\mathrm{CH}_{2}\right), 35.8\left(\mathrm{CH}_{2}\right), 70.8$ $(\mathrm{CH}), 114.1\left(=\mathrm{CH}_{2}\right), 115.0\left(=\mathrm{CH}_{2}\right), 125.1$ (aromat. $\left.\mathrm{CH}-\right), 125.7$ (aromat. $\left.\mathrm{CH}-\right), 126.5$ (aromat. $\mathrm{CH}-$ ), $138.2(=\mathrm{CH}-), 139.2(=\mathrm{CH}-), 143.7$ (quart. C), 173.1 (CO). El-MS m/z (rel. int.):348 ( $\left.\mathrm{M}^{+}, 2\right), 182$ (100), 97 (94). Elemental analysis: $\mathrm{C}_{21} \mathrm{H}_{32} \mathrm{O}_{2} \mathrm{~S}$ (348.55). Calcd.: C: 72.37. H: 9.25. S: 9.20. Found: C: 72.99. H: 9.78. S: 8.60.

\section{( \pm )-16-(Thiophen-2-yl)-oxacyclohexadec-11-en-2-one (4) (E/Z-mixture)}

$1.0 \mathrm{~g}(2.9 \mathrm{mmol})$ of 3 and $0.16 \mathrm{~g}(0.20 \mathrm{mmol})$ of Grubbs I catalyst (or $0.17 \mathrm{~g}(0.20 \mathrm{mmol})$ Grubbs II catalyst) were dissolved separately in $5 \mathrm{~mL}$ dry toluene each. Using two syringe pumps these solutions were simultaneously added dropwise to $250 \mathrm{~mL}$ of boiling dry toluene over a period of $12 \mathrm{~h}$ for Grubbs I and of $4 \mathrm{~h}$ for Grubbs II catalyst. The mixture was heated under reflux for another 12 hours under a $\mathrm{N}_{2}$-atmosphere for Grubbs I and $2 \mathrm{~h}$ 
for Grubbs II catalyst. Then the solvent was evaporated and the residue was purified by FCC (n-hexane/ ethyl acetate 9:1) to give $690 \mathrm{mg}$ (74\% for Grubbs I), $790 \mathrm{mg}$ (86 \% for Grubbs II) of 4 as a colourless oil. ${ }^{1} \mathrm{H}-\mathrm{NMR}\left(400 \mathrm{MHz}, \mathrm{D}_{6}\right.$-benzene): resonances of the predominating $E$-isomer: $\delta(\mathrm{ppm})=1.28\left(\mathrm{~m}, 10 \mathrm{H}, 5 \mathrm{CH}_{2}\right), 1.55\left(\mathrm{~m}, 4 \mathrm{H}, 2 \mathrm{CH}_{2}\right), 1.94(\mathrm{~m}$, $\left.4 \mathrm{H}, 2 \mathrm{CH}_{2}\right), 2.25\left(\mathrm{~m}, 4 \mathrm{H}, 2 \mathrm{CH}_{2}\right), 5.22(\mathrm{~m}, 2 \mathrm{H}, 2 \mathrm{CH}=$ containing a coupling constant of $\mathrm{J}=$ $15.3 \mathrm{~Hz}), 6.32(\mathrm{t}, \mathrm{J}=7.8 \mathrm{~Hz}, 1 \mathrm{H}, \mathrm{CH}), 6.90(\mathrm{dd}, \mathrm{J}=4.9 \mathrm{~Hz}, \mathrm{~J}=3.2 \mathrm{~Hz}, 1 \mathrm{H}$, aromat. $\mathrm{CH})$, $6.99(\mathrm{~d}, \mathrm{~J}=3.2 \mathrm{~Hz}, 1 \mathrm{H}$, aromat. $\mathrm{CH}), 7.20(\mathrm{~m}, 1 \mathrm{H}$, aromat. $\mathrm{CH}) .{ }^{13} \mathrm{C}-\mathrm{NMR}\left(400 \mathrm{MHz}, \mathrm{D}_{6}{ }^{-}\right.$ benzene): resonances of the predominating $E$-isomer: $\delta(\mathrm{ppm})=25.2\left(\mathrm{CH}_{2}\right), 25.6\left(\mathrm{CH}_{2}\right)$, $26.6\left(\mathrm{CH}_{2}\right), 27.4\left(\mathrm{CH}_{2}\right), 28.1\left(\mathrm{CH}_{2}\right), 28.2\left(\mathrm{CH}_{2}\right), 28.3\left(\mathrm{CH}_{2}\right), 32.0\left(\mathrm{CH}_{2}\right), 32.2\left(\mathrm{CH}_{2}\right), 34.9$ $\left(\mathrm{CH}_{2}\right), 35.7\left(\mathrm{CH}_{2}\right), 71.0(\mathrm{CH}), 125.4$ (aromat. $\left.\mathrm{CH}\right), 126.0$ (aromat. $\mathrm{CH}$ ), 126.5 (aromat. $\mathrm{CH}$ ), 130.5 (-CH=), 132.0 (-CH=), 143.3 (quart. C), 173.1 (CO). Cl-MS m/z (rel. int.): 320 $\left(\mathrm{M}^{+}, 15\right), 110$ (100). HR-MS: Calcd.: 320.1810. Found.: 320.1810. Elemental analysis: $\mathrm{C}_{19} \mathrm{H}_{28} \mathrm{O}_{2} \mathrm{~S}$ (320.50). Calcd.: C: 71.28. H: 8.81 S: 10.00. Found: C: 71.87. H: 9.08. S: 9.90.

\section{( \pm )-11,12-Dihydroxy-16-(thiophen-2-yl)-oxacyclohexadecan-2-one (5) (mixture of stereoisomers)}

$250 \mathrm{mg}(0.781 \mathrm{mmol})$ of lactone 4 were dissolved in $50 \mathrm{~mL}$ tert.-butanol/water (1:1) and $5.5 \mathrm{~g} \beta$-AD mix ${ }^{\circledR}$ (Aldrich) were added. The suspension was stirred for $12 \mathrm{~h}$ at room temperature. Then it was quenched with $100 \mathrm{~mL} 5 \%$ aqueous $\mathrm{Na}_{2} \mathrm{~S}_{2} \mathrm{O}_{3}$ solution and extracted with diethyl ether $(3 \times 30 \mathrm{~mL})$. The combined organic layers were dried over $\mathrm{Na}_{2} \mathrm{SO}_{4}$, the solvent was evaporated and the residue was purified by FCC (n-hexane/ethyl acetate $1: 1)$ to give $210 \mathrm{mg}(76 \%)$ of 5 as a colourless oil. ${ }^{1} \mathrm{H}-\mathrm{NMR}\left(400 \mathrm{MHz}, \mathrm{CDCl}_{3}\right): \delta$ $(\mathrm{ppm})=1.31\left(\mathrm{~m}, 14 \mathrm{H}, 7 \mathrm{CH}_{2}\right), 1.63\left(\mathrm{~m}, 4 \mathrm{H}, 2 \mathrm{CH}_{2}\right), 1.99\left(\mathrm{~m}, 2 \mathrm{H}, \mathrm{CH}_{2}\right), 2.34(\mathrm{t}, \mathrm{J}=7.2 \mathrm{~Hz}$, $\left.2 \mathrm{H}, \mathrm{CH}_{2}\right), 3.49(\mathrm{~m}, 2 \mathrm{H}, \mathrm{CH}), 5.24(\mathrm{~m}, 1 \mathrm{H}, \mathrm{CH}), 6.95(\mathrm{~m}, 2 \mathrm{H}, 2$ aromat. $\mathrm{CH}), 7.25(\mathrm{~m}, 1 \mathrm{H}$, aromat. $\mathrm{CH}) .{ }^{13} \mathrm{C}-\mathrm{NMR}\left(400 \mathrm{MHz}, \mathrm{CDCl}_{3}\right): \delta(\mathrm{ppm})=23.3-34.0\left(11 \mathrm{CH}_{2}\right), 73.6(\mathrm{CH}), 75.7$ $(\mathrm{CH}), 81.5(\mathrm{CH}), 123.1$ (aromat. $\mathrm{CH}), 124.4$ (aromat. $\mathrm{CH}$ ), 126.8 (aromat. $\mathrm{CH}$ ), 146.3 (quart. C), 179.4 (CO). MS (EI): $\mathrm{m} / \mathrm{z}(\%)=354\left(\mathrm{M}^{+}, 10\right), 336(5), 326(15), 168(27), 126$ (100), 97 (65). HR-MS: $\mathrm{C}_{19} \mathrm{H}_{30} \mathrm{O}_{4} \mathrm{~S}$ (354.51). HR-MS: Calcd.: 354.1865 Found: 354.1865.

\section{Acknowledgement}

We wish to thank Martina Stadler for her technical support. For the in vitro anti-cancer screening of compound $\mathbf{5}$ the authors thank the staff members of the National Cancer Institute ( $\mathrm{NCl})$, USA.

\section{Authors' Statement}

\section{Competing Interests}

The authors declare no conflict of interest.

\section{References}

[1] Mayer AMS, Rodríguez AD, Berlinck RGS, Hamann MT.

Marine pharmacology in 2005 - 6: Marine compounds with anthelminthic, antibacterial, anticoagulant, antifungal, antiinflammatory, antimalarial, antiprotozoal, antituberculosis, and antiviral activities; affecting the cardiovascular, immune and nervous systems, and other miscellaneous mechanisms of action.

Biochim Biophys Acta. 2009; 1790: 283-308.

http://dx.doi.org/10.1016/j.bbagen.20 09.03.011 
[2] Chin Y-W, Balunas M, Chai HB, Kinghorn AD.

Drug Discovery from Natural Sources.

AAPS Journal. 2006; 8: 239-253.

http://dx.doi.org/10.1208/aapsj080228

[3] Pal S.

A journey across the sequential development of macrolides and ketolides related to erythromycin. Tetrahedron. 2006; 62: 3171-3200.

http://dx.doi.org/10.1002/chin.200627242

[4] Erickson KL, Beutler JA, Cardellina JH, Boyd MR.

Salicylihalamides A and B, Novel Cytotoxic Macrolides from the Marine Sponge Haliclona sp.

J Org Chem. 1997; 62: 8188-8192.

http://dx.doi.org/10.1002/chin.199815264

[5] Logrado LP, Silveira D, Romeiro LAS, de Moraes MO, Cavalcanti BC, Costa-Lotufo LV, do O'Pessoa C, dos Santos ML.

Synthesis and biological evaluation of new salicylate macrolactones from anacardic acids.

J Braz Chem Soc. 2005; 16: 1217-1225.

http://dx.doi.org/10.1590/S0103-50532005000700020

[6] Eguchi T, Kobayashi K, Uekusa H, Ohashi Y, Kazutoshi M, Yoshitaka M, Kakinuma K. Stereostructure of a Novel Cytotoxic 18-Membered Macrolactone Antibiotic FD-891.

Org Lett. 2002; 4: 3383-3386.

http://dx.doi.org/10.1021/ol026518k

[7] Höfle G, Bedorf N, Steinmetz H, Schomburg D, Gerth K, Reichenbach H.

Epothilone A and B - Novel 16-Membered Macrolides with Cytotoxic Activity: Isolation, Crystal

Structure, and Conformation in Solution.

Angew Chem Int Ed. 1996; 35: 1567-1569.

http://dx.doi.org/10.1002/anie.199615671

[8] Schinzer D, Bourguet E, Ducki S.

Synthesis of Furano-Epothilone D.

Chem Eur J. 2004; 10: 3217-3224.

http://dx.doi.org/10.1002/chem.200400125

[9] Goodin S, Kane MP, Rubin EH.

Epothilones: Mechanism of Action and Biologic Activity.

J Clin Oncol. 2004; 22: 2015-2025.

http://dx.doi.org/10.1200/JCO.2004.12.001

[10] Alexander EJ, Rosa E, Bolos J, Castaner R.

Sagopilone.

Drugs Fut. 2008; 33: 496-505.

http://dx.doi.org/10.1358/dof.2008.033.06.1213136

[11] Mishra R, Tomar I, Singhal S, Jha KK.

Synthesis, properties and biological activity of thiophene: A review.

Der Pharma Chemica. 2011; 3: 38-54.

[12] Krauss J, Bracher F, Unterreitmeier D.

New Approach towards Cytotoxic Furyl Macrolactones by Ring Closing Metathesis.

Arch Pharm Chem Life Sci. 2006; 339: 629-632.

http://dx.doi.org/10.1002/ardp.200600135

[13] Schmidt C, Messmer R, Bracher F, Krauss J.

New Approach to Furan-Containing Macrolactones.

Turk J Chem. 2007; 31: 251-256.

[14] Krauss J, Unterreitmeier D.

Synthesis of New Lipophilic Ipomeanol Analogues and Their Cytotoxic Activities.

Arch Pharm Chem Life Sci. 2005; 338: 44-48.

http://dx.doi.org/10.1002/ardp.200400916 
[15] Krauss J, Unterreitmeier D, Antlsperger D.

Syntheses of 2,5-Dialkylfuran and Tetrahydrofuran Carbinols and Their Cytotoxic Activity.

Arch Pharm Pharm Med Chem. 2003; 336: 381-384.

http://dx.doi.org/10.1002/chin.200352106

[16] Gurjar MK, Nagaprasad R, Ramana CV, Karmakar S, Mohapatra DK.

Ring-closing metathesis mediated total synthesis of microcarpalide and herbarumin III.

ARKIVOC. 2005 part 3; 237-257.

[17] Straub BF.

Origin of the High Activity of Second-Generation Grubbs Catalysts.

Angew Chem Int Ed. 2005, 44: 5974-5978.

http://dx.doi.org/10.1002/anie.200501114

[18] Jacobsen EN, Marko I, Mungall WS, Schröder G, Sharpless KB.

Asymmetric Dihydroxylation via Ligand-Accelerated Catalysis.

J Am Chem Soc. 1988; 110: 1968-1970.

http://dx.doi.org/10.1021/ja00214a053

[19] Mosmann T.

Rapid Colorimetric Assay for Cellular Growth and Survival: Application to Proliferation and Cytotoxicity Assays.

J Immunol Methods. 1983; 65: 55-63.

http://dx.doi.org/10.1016/0022-1759(83)90303-4

[20] Lipinski CA, Lombardo F, Dominy BW, Feeney PJ.

Experimental and computational approaches to estimate solubility and permeability in drug discovery and development settings.

Adv Drug Deliv Rev. 2001; 46: 3-26.

http://dx.doi.org/10.1016/S0169-409X(00)00129-0 